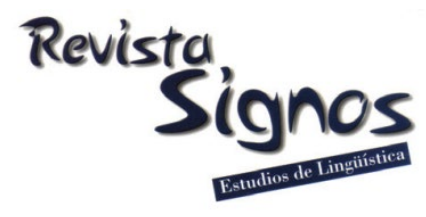

\title{
Relación entre vocabulario y comprensión lectora: Un estudio transversal en educación básica
}

\section{Relationship between vocabulary and reading comprehension: $A$ transversal study in primary education}

\author{
Susana Figueroa Sepúlveda \\ UNIVERSIDAD ARTURO PRAT \\ CHILE \\ susfigue@unap.cl
}

\author{
José Luis Gallego Ortega \\ UNIVERSIDAD DE GRANADA \\ ESPAÑA \\ jlgalleg@ugr.es
}

Recibido: 04-III-2020 / Aceptado: 05-VIII-2020

DOI: $10.4067 /$ S0718-09342021000200354

\section{Resumen}

En la actualidad, la lectura se percibe como una actividad cognitiva compleja. De ahí que la lectura se convierta con relativa frecuencia en un auténtico desafío para alumnos y profesores. En este sentido, el objetivo de esta investigación fue conocer el nivel de desarrollo léxico y de comprensión lectora de los estudiantes chilenos de educación básica, para analizar la correlación entre el vocabulario y la comprensión lectora, y su comportamiento según género, capacidad cognitiva, nivel escolar (curso), ciclo y tipo de centro. Para este estudio, se trabajó con una muestra de 404 estudiantes chilenos escolarizados en tres escuelas urbanas de una misma localidad, aunque de distinta titularidad: pública (municipal), concertada (particular subvencionada) y privada (particular pagada), que pertenecían a su vez a distintos niveles socioeconómicos (medio-bajo, medio y alto, respectivamente). Mediante un estudio de tipo descriptivo y correlacional se desveló el nivel de comprensión lectora y de vocabulario, así como las diferencias entre escolares y las correlaciones existentes, según las distintas variables. Se concluyó que el vocabulario, el nivel escolar, el tipo de centro y la capacidad cognitiva de los escolares correlacionan significativamente con la comprensión lectora, sin que la variable género haya sido determinante en ningún caso.

Palabras Clave: Lectura, vocabulario, comprensión lectora, educación básica, desarrollo lector. 


\begin{abstract}
Currently, reading is perceived as a complex cognitive activity. Hence reading becomes relatively often a real challenge for students and teachers. In this sense, the objective of this research was to determine the level of vocabulary development and reading comprehension of Chilean elementary school students, to analyze the correlation between vocabulary and reading comprehension, and their behavior according to gender, cognitive ability, school level (school year they were attending), stage (from 1st to 4 th and 5 th to 8 th grade) and kind of school. For this study, we worked with a sample of 404 Chilean students enrolled in three urban schools in the same town, but different ownership: public (municipal), concerted (particularly subsidized) and private, who belonged to different socioeconomic levels (medium-low, medium and high, respectively). Through a descriptive and correlational study the level of reading comprehension and vocabulary was unveiled, as well as the differences between students and the existing correlations, according to the different variables. It was concluded that vocabulary, school level, school type and cognitive ability of the students are correlated significantly with reading comprehension, without the gender variable being decisive in any case.
\end{abstract}

Key Words: Reading, vocabulary, reading comprehension, primary education, reading development.

\title{
INTRODUCCIÓN
}

La lectura se sitúa entre las habilidades más trascendentales de cualquier idioma. La preocupación por la lectura es una constante entre profesionales e investigadores de cualquier país y su enseñanza representa un gran reto para la escuela de todos los tiempos. Leer es algo más que acceder al reconocimiento visual de palabras (Carrillo \& Alegría, 2015) y decodificar el vocabulario de un texto mediante la activación de habilidades fonológicas (Bohórquez, Cabal \& Quijano, 2014), es liberar estrategias cognitivas que facultan al lector para comprender globalmente los significados del texto (Perfetti, 2007).

En la actualidad, la lectura se percibe como un instrumento esencial para la construcción de significados, responsable, en buena medida, del aprendizaje escolar. Una vez adquirida, se convierte en una herramienta clave para el acopio de nuevos conocimientos y la conquista del éxito académico (Willems, Jansma, Blomert \& Vaessen, 2016). Pero si se admite que el fin último de un proceso lector es la comprensión de un texto, la lectura se torna en una actividad cognitiva compleja en la que el lector, activando una amplia gama de procesos, ha de inferir los significados que encierra el texto para construir la información que éste contiene de forma más o menos explícita.

Desde esta perspectiva, la lectura es un proceso constructivo, activo, situacional y estratégico (Alliende \& Condemarín, 2002; Schelling, Aarnoutse \& Leeuwe, 2006), orientado a la comprensión textual, la cual se vincula no solo con el reconocimiento que hace el lector de las ideas del texto sino con sus habilidades lingüísticas, su actitud 
e intenciones, sus conocimientos previos y su misma capacidad lectora (Molina, 2008; Lara, Gómez, García, Guerrero \& Niño, 2010).

La investigación sobre la lectura ha proporcionado información valiosa y detallada sobre el desarrollo de las habilidades de comprensión y los factores que influyen y están influenciados por estas habilidades (McNamara \& Magliano, 2009). De ahí que los problemas de los escolares con la lectura no sean nuevos y tampoco el interés por conocer las causas que los generan. Evaluaciones nacionales e informes internacionales como SIMCE (Sistema de Medición de la Calidad de la Educación), PISA (Programme for International Student Assessment) o PIRLS (Progress in International Reading Literacy Study) han evidenciado los bajos rendimientos lectores del alumnado, poniendo de manifiesto los problemas de comprensión lectora de la población escolar.

En Chile, los últimos resultados nacionales en lectura evidencian aún datos inquietantes, a tenor del nivel de aprendizaje alcanzado por los escolares. Según el SIMCE (2018), en $4^{\circ}$ básico, el nivel de los estándares de aprendizaje son los siguientes: 29\% (insuficiente), 26,3\% (elemental), 44,7\% (adecuado). Y en $6^{\circ}$ básico: $37,3 \%$ (insuficiente), 31,2\% (elemental), 31,5\% (adecuado), por lo que la comprensión lectora se mantiene como un auténtico desafío para el sistema educativo chileno. En este sentido, la investigación que se presenta trata de explorar esta problemática desde la dimensión del vocabulario. En efecto, numerosos estudios han puesto de manifiesto el papel que el vocabulario desempeña en el proceso lector. Muter, Hulme, Snowling y Stevenson (2004) han demostrado que el conocimiento del vocabulario y el reconocimiento de palabras son determinantes para la comprensión lectora. Equivalentemente, otros investigadores han encontrado una marcada correlación entre la comprensión lectora y el nivel de vocabulario (Suárez, Moreno \& Godoy, 2010; Riffo, Caro \& Sáez, 2018), o una relación directa entre la comprensión y el léxico pasivo que posee un lector (Cepeda, Granada \& Pomes, 2014).

En esta misma línea, diferentes investigadores han considerado el vocabulario como un predictor relevante de la comprensión en los primeros años de escolaridad (Lonigan, Burgess \& Anthony, 2000; Perfetti, 2010; López \& Rubio, 2014), admitiendo que la riqueza léxica no solo beneficia el aprendizaje escolar sino que incrementa la comprensión textual (Hirsch, 2007; Villalonga, Padilla \& Burin, 2014), o que el repertorio léxico subyacente promueve la asociación entre la comprensión lectora y la lectura de palabras (Nation \& Snowling, 1998). El aprendizaje del vocabulario es crucial para la adquisición del lenguaje en general y de la lectura, en particular, de manera que cuando los estudiantes desconocen numerosas palabras de su idioma se incrementan las dificultades a la hora de procesar un texto. Por ello, se sabe que muchos de los problemas de comprensión lectora incluyen el reconocimiento de palabras y el acceso al léxico (Moghadam, Zainal \& Ghaderpour, 2012). 
Ricketts, Nation y Bishop (2007) estudiaron qué habilidades lectoras se relacionaban con el vocabulario y atribuyeron al léxico un papel fundamental, considerándolo como un predictor importante de la comprensión textual. Efectivamente, las diferencias individuales en el vocabulario desempeñan un papel relevante en los procesos lectores (Muter et al., 2004; Nation \& Snowling, 2004), de manera que las dificultades de comprensión pueden ser atribuidas a un insuficiente desarrollo léxico (Canet, Burin, Andrés \& Urquijo, 2013). Según Nagy y Scott (2000), los estudios sobre el desarrollo léxico y su efecto en la comprensión sostienen que el lector debe conocer en torno al 95\% de las palabras que contiene el texto para poder comprenderlo correctamente. Solo así se podría acceder al significado global del texto e inferir el significado de las palabras menos conocidas (Hirsch, 2003).

Otras investigaciones, realizadas en diferentes contextos (Nation, Cocksey, Taylor \& Bishop, 2010; González \& Castro, 2016; Swart, Muijselaar, Steenbeek, Droop, Jong \& Verhoeven, 2017), analizaron la correlación entre el vocabulario y la comprensión y observaron que los estudiantes con dificultades de comprensión lectora exhibían un vocabulario más pobre que aquellos que mostraban una buena comprensión, advirtiendo una relación de reciprocidad entre el vocabulario y la comprensión lectora. Equivalentemente, los datos del estudio de Verhoeven y Perfetti (2011) han subrayado que_el conocimiento de las formas y los significados de las palabras predicen el desarrollo de la comprensión lectora, respaldando la hipótesis de la calidad léxica. Igualmente se ha señalado que los procesos de significado de palabras son componentes causales de la comprensión (Perfetti \& Stafura, 2014). Se diría que el vocabulario hace contribuciones únicas y significativas a la predicción de la capacidad de comprensión (Oakhill \& Cain, 2012).

Asimismo, diferentes estudios han confirmado que las variables predictoras de la comprensión lectora intervienen de manera interconectada, y consideran que, junto con el vocabulario, también es determinante la escolaridad de los padres y el nivel socioeconómico, al hallar una correlación positiva entre estas variables (Morales, 2011; Cuadro \& Balbi, 2012; Fonseca, Pujals, Lasala, Lagomarsino, Migliardo, Aldrey, Buonsanti \& Barreyro, 2014; Urquijo, García \& Fernandes, 2015; Sousa \& Hübner, 2017).

Igualmente, de forma reiterada, la investigación ha revelado el papel discriminador que ejerce el vocabulario para diferenciar entre malos y buenos lectores, dado que los lectores noveles y los lectores ineficientes carecen de las habilidades necesarias para el análisis de palabras (Coelho \& Correa, 2017). Este relevante papel atribuido al vocabulario no impide que algunos estudios hayan demostrado que la incidencia del vocabulario en la comprensión lectora ha sido menor de la esperada (Muter et al., 2004; Salvador, Gallego \& Mieres, 2007). Por ello, parece razonable analizar realidades escolares concretas para determinar cuáles son las variables condicionantes de la 
lectura a fin de elaborar y desarrollar programas específicos que permitan paliar o superar las dificultades iniciales de los estudiantes para que éstos puedan alcanzar un mayor desempeño lector.

Desde esa perspectiva, esta investigación se propuso estudiar la contribución del vocabulario al desempeño de la comprensión lectora en escolares de enseñanza básica, para determinar la asociación entre estas variables, y su comportamiento, según tipo de centro, género y capacidad cognitiva del alumnado, nivel escolar y ciclo.

\section{Método}

Se trata de una investigación empírica no experimental, en la que se utilizó una estrategia descriptiva (observacional) y otra asociativa, para explorar la relación existente entre variables (Ato, López \& Benavente, 2013).

\subsection{Participantes}

Se trabajó con una muestra intencional de 404 estudiantes (Tabla 1) de los cuales 212 son niñas (52,5\%) y 192 niños (47,5\%), que estaban escolarizados en tres escuelas de Iquique (Chile) de distinta titularidad: municipal (pública), particular subvencionada (concertada) y particular pagada (privada), y pertenecían a distintos niveles socioeconómicos (NSE). Según el Sistema de Medición de la Calidad de la Educación (SIMCE, 2018), los NSE de las escuelas son: medio-bajo, medio y alto, respectivamente. Se excluyeron de la muestra los estudiantes que repetían curso escolar.

Tabla 1. Muestra global.

\begin{tabular}{|l|c|c|c|c|c|c|c|c|c|c|c|c|c|c|}
\hline \multicolumn{1}{|c|}{ Colegio } & \multicolumn{4}{|c|}{$\mathbf{2}^{\mathbf{o}}$} & \multicolumn{4}{c|}{$\mathbf{4}^{\mathbf{0}}$} & \multicolumn{3}{c|}{$\mathbf{6}^{\mathbf{0}}$} & \multicolumn{4}{c|}{$\mathbf{8}^{\mathbf{0}}$} & Total \\
\hline & Niñas & Niños & Total & Niñas & Niños & Total & Niñas & Niños & Total & Niñas & Niños & Total & \\
\hline Privado & 26 & 17 & 43 & 26 & 22 & 48 & 23 & 24 & 47 & 23 & 14 & 37 & 175 \\
\hline Concertado & 17 & 14 & 31 & 17 & 12 & 29 & 18 & 12 & 30 & 13 & 15 & 28 & 118 \\
\hline Público & 9 & 15 & 24 & 14 & 17 & 31 & 13 & 18 & 31 & 13 & 12 & 25 & 111 \\
\hline Totales & 52 & 46 & 98 & 57 & 51 & 108 & 54 & 54 & 108 & 49 & 41 & 90 & 404 \\
\hline Promedio Edad & 7,7 & 7,7 & 7,7 & 9,7 & 9,7 & 9,7 & 11,5 & 11,8 & 11,6 & 13,8 & 13,5 & 13,7 & 10,6 \\
\hline DT & 0,42 & 0,58 & 0,54 & 0,50 & 0,50 & 0,52 & 0,33 & 0,75 & 0,65 & 0,75 & 0,58 & 0,70 & 2,25 \\
\hline
\end{tabular}

\subsection{Variables}

En el diseño de investigación, se han considerado variables independientes (vocabulario, tipo de centro, nivel escolar, ciclo, capacidad intelectual y género de los estudiantes), y la comprensión lectora como variable dependiente.

\subsection{Instrumentos}

Los datos se han recabado mediante los siguientes instrumentos de medida: 
1) Test de Matrices Progresivas (Raven, Court \& Raven, 2014). Es una prueba no verbal, validada en Chile, que puede administrarse de forma individual o colectiva. Mide la capacidad intelectual por medio de la comparación de formas y el razonamiento por analogías. Habitualmente se utiliza para formar grupos de capacidad intelectual homogénea y para detectar alumnos con discapacidad. Según los resultados, los sujetos son clasificados por rangos: Rango I (intelectualmente superior, si su puntaje es $\geq$ a 95 para los sujetos de su edad); Rango II (definidamente superior al término medio: $\geq 75 \mathrm{y}<95$ ). Rango III (intelectualmente término medio: $<75 \mathrm{y}>25$ ). Rango IV (decididamente inferior al término medio: $\geq 25$ y $<5$ ). Rango $V$ (intelectualmente deficiente: $\leq 5)$.

2) Prueba de Comprensión Lectora de Complejidad Lingüística Progresiva (Alliende, Condemarín \& Milicic, 2012). Validada en Chile, mide la comprensión general por medio de frases y textos. Se presenta en 8 niveles de lectura donde cada nivel va adquiriendo una dificultad creciente desde el punto de vista lingüístico, produciéndose paralelamente un incremento en la dificultad de comprensión. Cada nivel corresponde a un grado escolar. La tarea consiste en leer silenciosamente y contestar a preguntas de elección múltiple.

3) TEVI-R test de vocabulario en imágenes (Echeverría, Herrera \& Segure, 2002). Este test, validado en Chile, mide el vocabulario pasivo del sujeto. Éste ha de señalar una de las cuatro imágenes de una lámina, que se corresponde con la palabra-estímulo pronunciada por el evaluador. Se puede administrar de forma individual con niños menores de 8 años y de forma colectiva a niños de más edad. Según el puntaje, se subordina al estudiante en una escala de categorías (sobresaliente, muy bueno, normal, retraso leve o retraso grave).

\subsection{Procedimiento}

Inicialmente, se expuso a los directores de las escuelas los objetivos y características del estudio para obtener su autorización. Asimismo, se informó a los profesores de la asignatura de Lenguaje de los niveles implicados. Posteriormente, se consensuaron fechas y horarios para la aplicación de las pruebas en la sala de clase, tarea que se realizó durante un mes en el año escolar de 2017. Se contó con la ayuda de seis estudiantes de Psicopedagogía, próximas a egresar y previamente entrenadas. El test de Matrices Progresivas fue aplicado de forma colectiva en todos los cursos. La prueba de comprensión lectora se aplicó por el profesorado de la asignatura, con el apoyo de las estudiantes universitarias. La prueba de vocabulario se aplicó individualmente a los escolares de segundo nivel y de manera colectiva al resto. Siempre se mostró un ejemplo para asegurar la comprensión de los escolares.

Todos los alumnos de la muestra accedieron voluntariamente a participar en el estudio, en el que se respetaron los valores éticos de una investigación de estas 
características (protección de datos, confidencialidad, aprobación por las autoridades escolares y consentimiento informado).

\subsection{Análisis estadístico}

Se utilizó la estadística descriptiva para obtener frecuencias, medias y desviaciones estándar. Se recurrió a la $t$ de Student para analizar la significatividad de las diferencias entre medias según género y ciclo, utilizando el método de bootstrap en caso de muestras no normales. Se empleó la F-Snedecor (ANOVA de un factor) para muestras independientes, para comparar las medias de los colegios y cursos, y el test de Brown-Forsythe, cuando no se cumplían los principios de normalidad y homocedasticidad de los datos. Se utilizó la prueba de Chi-cuadrado para testar la hipótesis de igualdad de distribución de resultados cualitativos según género, nivel escolar y colegio, así como para establecer el contraste de igualdad de distribución de resultados por género en cada curso y colegio, y también para estudiar las diferencias entre colegios por curso.

Finalmente, se realizó una correlación bivariada ( $\varrho$ de Spearman) para describir la asociación entre vocabulario y comprensión lectora, y se efectuó una regresión múltiple (o multivariable) para estudiar la asociación global de la puntuación obtenida en vocabulario con los porcentajes de acierto en comprensión lectora, ajustados por tipo de colegio, nivel escolar y clasificación del alumnado.

Los análisis se realizaron con la ayuda del software IBM SPSS v.19, y, como criterio de significación, se fijó un valor alpha menor de .05.

\section{Resultados}

A continuación, se presentan los resultados del análisis descriptivo y bivariante, así como los resultados del análisis de regresión múltiple realizado para obtener las conclusiones finales y responder a los objetivos de esta investigación.

\subsection{Resultados en comprensión lectora}

Como se observa en la Tabla 2, la media de las puntuaciones obtenida por los niños y las niñas en comprensión lectora es muy similar, no habiendo diferencias significativas $(p=.298)$. Por colegios, fueron los estudiantes del centro privado quienes obtuvieron el valor más elevado $(\overline{\boldsymbol{x}}=68,91 \%)$, seguidos del concertado $(\overline{\boldsymbol{x}}=56,16 \%)$ y el público $(\overline{\boldsymbol{x}}=46,62 \%)$, siendo estas diferencias significativas $(p<.001)$. Según el ciclo, existen diferencias significativas a favor del primero $(p=.001)$. Al considerar los resultados por nivel escolar (curso), el análisis reflejó diferencias significativas $(p<.001)$, observándose una tendencia decreciente según aumenta el nivel de escolarización, con una brecha cercana al $20 \%$ entre $2^{\circ}$ y $8^{\circ}$. 
Tabla 2. Resultados globales en comprensión lectora según género, colegio, ciclo y curso.

\begin{tabular}{|c|c|c|c|c|c|c|c|}
\hline & & \multirow{2}{*}{$\mathbf{N}$} & \multirow{2}{*}{$\bar{x}$} & \multirow{2}{*}{ D'T } & \multicolumn{2}{|c|}{ Intervalo de Confianza 95\% } & \multirow{2}{*}{ p-valor } \\
\hline & & & & & Inferior & Superior & \\
\hline \multirow{2}{*}{ Género } & Niños & 192 & 57.85 & 22.05 & 54.46 & 60.84 & \multirow{2}{*}{$.298^{a}$} \\
\hline & Niñas & 212 & 60.16 & 21.49 & 57.25 & 63.02 & \\
\hline \multirow{3}{*}{ Colegios } & Privado & 175 & 68.91 & 18.14 & 66.24 & 71.45 & \multirow{3}{*}{$<.001^{\mathrm{c}}$} \\
\hline & Concertado & 118 & 56.16 & 21.77 & 52.05 & 60.09 & \\
\hline & Público & 111 & 46.62 & 19.76 & 43.17 & 50.14 & \\
\hline \multirow{2}{*}{ Ciclos } & $1^{\circ}$ & 206 & 65.18 & 24.00 & 61.87 & 68.56 & \multirow{2}{*}{$.001 \mathrm{a}$} \\
\hline & $2^{\circ}$ & 198 & 52.69 & 17.02 & 50.39 & 55.14 & \\
\hline \multirow{4}{*}{ Niveles } & $2^{\circ}$ básico & 98 & 70.01 & 27.15 & 64.44 & 75.08 & \multirow{4}{*}{$<.001^{\mathrm{b}}$} \\
\hline & $4^{\circ}$ básico & 108 & 60.80 & 19.86 & 56.97 & 64.74 & \\
\hline & $6^{\circ}$ básico & 108 & 53.33 & 18.58 & 49.93 & 56.52 & \\
\hline & $8^{\circ}$ básico & 90 & 51.92 & 15.00 & 48.61 & 55.25 & \\
\hline
\end{tabular}

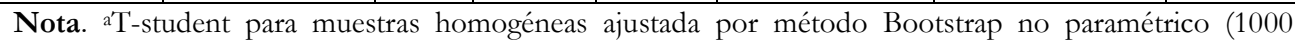
replicaciones) para muestras no normales. ${ }^{b}$ Brown-Forsythe para muestras no normales. ${ }^{c}$ F-Snedecor para muestras homogéneas

Los análisis post-hoc (HSD Tukey) han corroborado los resultados anteriores. En efecto, el centro privado obtiene resultados significativamente mejores que los otros dos colegios $(p<.001)$. Además el centro concertado logró mejores resultados que el colegio público $(p=.001)$, siendo éste el que lograra los resultados más bajos respecto de los anteriores (centro privado: $p<.001$; centro concertado: $p=.001$ ). Por niveles, el alumnado de $2^{\circ}$ básico logró los mejores resultados, que fueron significativos con respecto a los demás niveles analizados $(p<.05)$. A su vez, los resultados de los escolares de $4^{\circ}$ han sido significativamente mejores que los de $6^{\circ}$ y $8^{\circ}$ básico $(p<.05)$. No se observaron diferencias significativas entre $6^{\circ}$ y $8^{\circ}$ básico $(p>.05)$.

\subsection{Resultados en vocabulario}

El análisis de los datos (Tabla 3) reveló la inexistencia de diferencias significativas por género $(p=.497)$, siendo las puntuaciones medias de los niños y las niñas bastante similares. Al comparar los colegios, sin embargo, se observó en las puntuaciones medias de vocabulario diferencias significativas $(\phi<.001)$. De hecho, se halló el valor de la media más elevado en el colegio privado (48.78), seguido del público (44.41) y el concertado (42.37). Asimismo, se detectaron diferencias significativas por ciclo $(p=.001)$ y curso $(p<.001)$, observándose una tendencia creciente según aumenta el ciclo o el curso, exceptuando en $8^{\circ}$ básico. 
Tabla 3. Resultados globales de vocabulario según género, colegio, ciclo y curso.

\begin{tabular}{|c|c|c|c|c|c|c|c|}
\hline & & \multirow{2}{*}{$\mathbf{N}$} & \multirow{2}{*}{$\bar{x}$} & \multirow{2}{*}{ DT } & \multicolumn{2}{|c|}{ Intervalo de Confianza 95\% } & \multirow{2}{*}{ p-valor } \\
\hline & & & & & Inferior & Superior & \\
\hline \multirow{2}{*}{ Género } & Niños & 192 & 46.11 & 11.26 & 44.51 & 47.59 & \multirow{2}{*}{$.497 a$} \\
\hline & Niñas & 212 & 45.34 & 12.03 & 43,63 & 46.94 & \\
\hline \multirow{3}{*}{ Colegios } & Privado & 175 & 48.78 & 12.85 & 46.86 & 50.59 & \multirow{3}{*}{$<.001^{b}$} \\
\hline & Concertado & 118 & 42.37 & 9.04 & 40.86 & 43.95 & \\
\hline & Público & 111 & 44.41 & 11.02 & 42.47 & 46.53 & \\
\hline \multirow{2}{*}{ Ciclos } & $1^{\circ}$ & 206 & 43.02 & 11.02 & 41.61 & 44.56 & \multirow{2}{*}{$.001^{\mathrm{a}}$} \\
\hline & $2^{\circ}$ & 198 & 48.49 & 11.69 & 46.91 & 50.21 & \\
\hline \multirow{4}{*}{ Niveles } & $2^{\circ}$ básico & 98 & 39.98 & 11.31 & 37.81 & 42.30 & \multirow{4}{*}{$<.001^{b}$} \\
\hline & $4^{\circ}$ básico & 108 & 45.79 & 10.02 & 43.96 & 47.69 & \\
\hline & $6^{\circ}$ básico & 108 & 51.83 & 12.04 & 49.64 & 54.09 & \\
\hline & $8^{\circ}$ básico & 90 & 44.49 & 9.92 & 42.42 & 46.64 & \\
\hline
\end{tabular}

Nota. ${ }^{2}$ T-student para muestras homogéneas ajustada por método Bootstrap no paramétrico (1000 replicaciones) para muestras no normales. ${ }^{b}$ Brown-Forsythe para muestras no normales.

Los datos del análisis post-hoc (HSD Tukey) confirmaron que los resultados del centro privado son significativamente mejores que los obtenidos por los otros dos colegios $(p<.05)$. Las diferencias entre los colegios concertado y público no fueron significativas ( $p>$.05). Igualmente, se constató que los resultados de $2^{\circ}$ básico fueron significativamente menores que los de $4^{\circ}, 6^{\circ}$ y $8^{\circ}$ curso $(p<.05)$. Así también, los escolares de $4^{\circ}$ básico obtienen menores logros que los de $6^{\circ}$ curso $(p<.001)$. Sin embargo, los estudiantes de $6^{\circ}$ básico consiguen mejores resultados que los de $8^{\circ}$ básico $(p<.001)$.

No obstante, las puntuaciones obtenidas en el test de vocabulario se clasificaron en categorías con el propósito de poder simplificar y agrupar a los estudiantes y ver las diferencias entre ellos (Tabla 4). Si se considera la muestra global, las niñas obtuvieron un porcentaje más elevado que los niños en las categorías de retraso leve o grave, muy bueno y sobresaliente, mientras que los niños superaron en porcentaje a las niñas en la categoría normal, siendo estas diferencias entre niños y niñas significativas $(p=.017)$. Pero si se reagrupan las categorías sobresaliente, muy bueno y normal, los niños superaron a las niñas $(55.2 \%$ versus $43,4 \%)$. Por colegios, también se hallaron diferencias significativas $(p<.001)$. En el colegio privado se observó el porcentaje más elevado de alumnos con resultados sobresaliente y muy bueno, mientras que en el colegio público los escolares obtuvieron un mayor porcentaje en la categoría retraso grave. El mayor porcentaje de alumnos con retraso leve se detectó en el colegio concertado.

Asimismo, se hallaron diferencias significativas entre los ciclos $(p<.001)$. En el segundo ciclo se registró un mayor porcentaje de estudiantes en las categorías de sobresaliente, muy bueno y normal, mientras que en el primer ciclo los valores más elevados se observaron en retraso leve o grave. Del mismo modo, se descubrieron diferencias significativas entre niveles (cursos) $(p<.001)$. En $6^{\circ}$ curso se encontraron más alumnos en las categorías sobresaliente y muy bueno que en los demás cursos; en 
$8^{\circ}$ un mayor porcentaje de alumnos con resultado normal; en $4^{\circ}$ un mayor porcentaje de alumnos con retraso leve y en $2^{\circ}$ curso un porcentaje más elevado de alumnos con retraso grave.

Tabla 4. Distribución del alumnado según test de vocabulario, por género, colegio, ciclo y nivel.

\begin{tabular}{|c|c|c|c|c|c|c|c|c|c|c|c|c|}
\hline & & \multicolumn{2}{|c|}{ Sobresa-liente } & \multicolumn{2}{|c|}{ Muy bueno } & \multicolumn{2}{|c|}{ Normal } & \multicolumn{2}{|c|}{$\begin{array}{l}\text { Retraso } \\
\text { leve }\end{array}$} & \multicolumn{2}{|c|}{$\begin{array}{l}\text { Retraso } \\
\text { grave }\end{array}$} & \multirow[b]{2}{*}{ P-valor } \\
\hline & & $\mathbf{N}$ & $\%$ & $\mathbf{N}$ & $\%$ & $\mathbf{N}$ & $\%$ & $\mathbf{N}$ & $\%$ & $\mathbf{N}$ & $\%$ & \\
\hline \multirow{2}{*}{ Género } & Niños & 11 & 5.7 & 29 & 15.1 & 66 & 34.4 & 61 & 31.8 & 25 & 13.0 & \multirow{2}{*}{$.017^{\mathrm{a}}$} \\
\hline & Niñas & 14 & 6.6 & 37 & 17.5 & 41 & 19.3 & 82 & 38.7 & 38 & 17.9 & \\
\hline \multirow{3}{*}{ Colegios } & Privado & 19 & 10.9 & 45 & 25.7 & 37 & 21.1 & 53 & 30.3 & 21 & 12.0 & \multirow{3}{*}{$<.001^{\mathrm{a}}$} \\
\hline & Concertado & 1 & 0.8 & 7 & 5.9 & 36 & 30.5 & 53 & 44.9 & 21 & 17.8 & \\
\hline & Público & 5 & 4.5 & 14 & 12.6 & 34 & 30.6 & 37 & 33.3 & 21 & 18.9 & \\
\hline \multirow{2}{*}{ Ciclos } & $1^{\circ}$ & 10 & 4.9 & 19 & 9.2 & 52 & 25.2 & 86 & 41.7 & 39 & 18.9 & \multirow{2}{*}{$<.001^{\mathrm{a}}$} \\
\hline & $2^{\circ}$ & 15 & 7.6 & 47 & 23.7 & 55 & 27.8 & 57 & 28.8 & 24 & 12.1 & \\
\hline \multirow{4}{*}{ Niveles } & $2^{\circ}$ básico & 4 & 4.1 & 5 & 5.1 & 21 & 21.4 & 40 & 40.8 & 28 & 28.6 & \multirow{4}{*}{$<.001^{\mathrm{a}}$} \\
\hline & $4^{\circ}$ básico & 6 & 5.6 & 14 & 13.0 & 31 & 28.7 & 46 & 42.6 & 11 & 10.2 & \\
\hline & $6^{\circ}$ básico & 14 & 13.0 & 34 & 31.5 & 24 & 22,2 & 29 & 26.9 & 7 & 6.5 & \\
\hline & $8^{\circ}$ básico & 1 & 1.1 & 13 & 14.4 & 31 & 34.4 & 28 & 31.1 & 17 & 18.9 & \\
\hline
\end{tabular}

Nota. a Chi-cuadrado de Pearson

Según la información derivada del cálculo de los residuos tipificados corregidos, las niñas tienen mayor porcentaje en retraso leve, retraso grave, muy bueno y sobresaliente, mientras que los niños tienen mayor porcentaje en la categoría de normal. En este caso, se pudo comprobar la relación entre las variables género y vocabulario, dado el p-valor (significación asintótica bilateral) obtenido por la Chicuadrado de Pearson $\left(\chi^{2}=11,977 \mathrm{gl} 4 p=.018\right)$. Asimismo, se pudo observar que el colegio privado concentra en las categorías muy bueno y sobresaliente el mayor porcentaje, mientras que el colegio concertado lo consigue en retraso leve y normal, y el colegio público en retraso grave y normal. Equivalentemente, se pudo constatar que el tipo de colegio y nivel de vocabulario están relacionados $\left(\chi^{2}=40,670 \mathrm{gl} 8 p=.000\right)$. Igualmente, se puede indicar que los estudiantes de primer ciclo presentan mayor porcentaje en retraso leve y retraso grave, mientras que los estudiantes del segundo demuestran mayor porcentaje en muy bueno, sobresaliente y normal. La significación asintótica obtenida $\left(\chi^{2}=22,266 \mathrm{gl} 4 p=.000\right)$ permite concluir que las variables están relacionadas de manera dependiente. Finalmente, en el nivel escolar se comprobó que en retraso grave son los estudiantes de $2^{\circ}$ básico quienes presentan mayor porcentaje, en retraso leve son los de $4^{\circ}$ cuarto curso, los estudiantes de $8^{\circ}$ básico lo alcanzan en la categoría de normal, y los de $6^{\circ}$ curso en las categorías de muy bueno y sobresaliente. También ahora la significación asintótica obtenida muestra relación de dependencia entre el nivel de vocabulario y el curso escolar $\left(\chi^{2}=64,332 \mathrm{gl} 12 p=.000\right)$.

Los resultados de las pruebas de Chi-cuadrado se presentan en el siguiente gráfico de mosaico (Figura 1). 


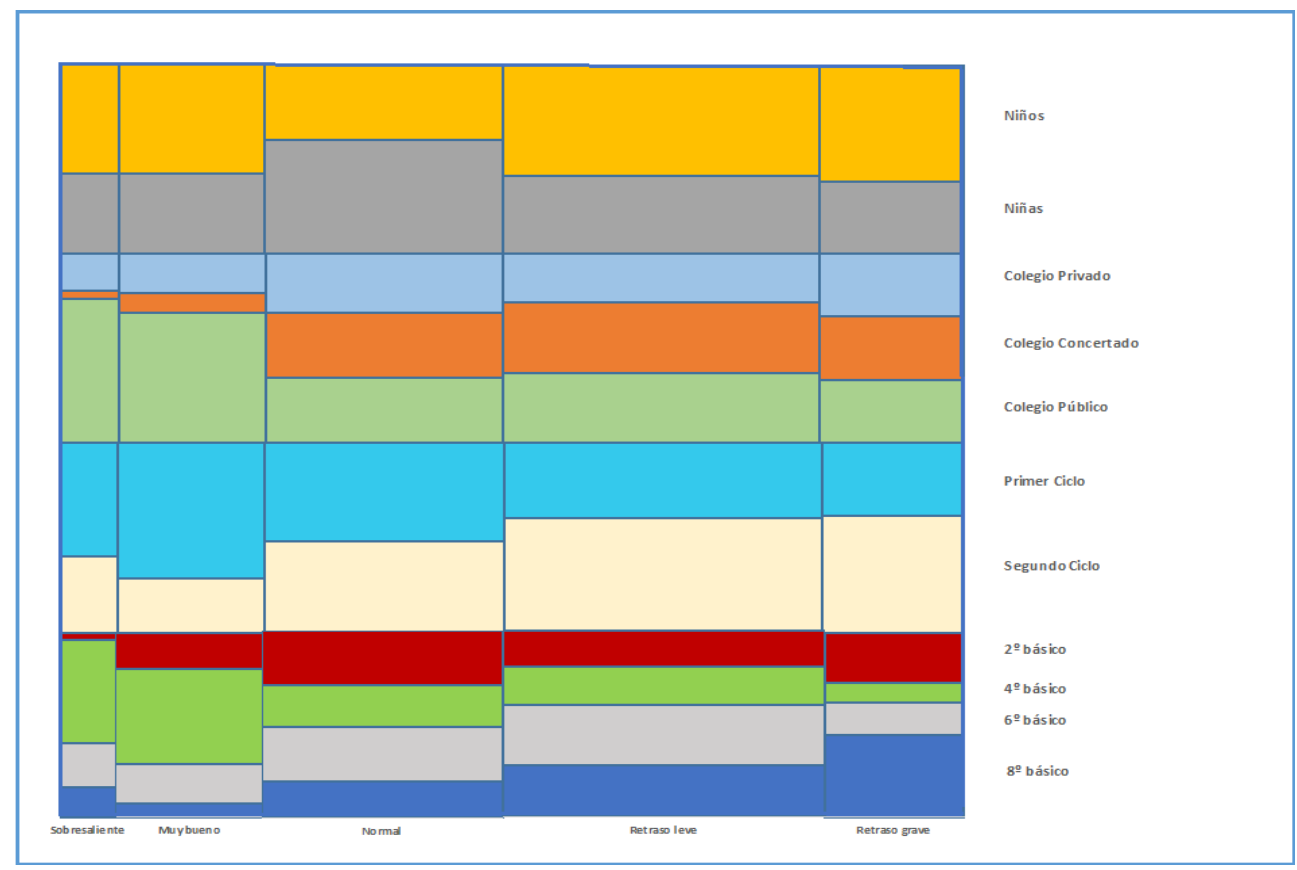

Figura 1. Resultados de las pruebas de Chi-cuadrado.

\subsection{Resultados de la asociación entre el vocabulario y la compresión lectora}

En la Tabla 5, se muestran los resultados del análisis bivariante, con técnica no paramétrica Spearman, dado que no se cumplía el supuesto de normalidad de los datos en todos los grupos de desglose. Considerando la muestra global, se observó una asociación significativa entre el vocabulario y la comprensión lectora $(\varrho=.155, p=.002)$. También se apreció correlación entre las alumnas $(\varrho=.227, p=.001)$ y entre el alumnado de $2^{\circ}$ ciclo $(\varrho=.374, p=<.001)$, así como en los niveles de $2^{\circ}(\varrho=.290$, $p=.004), 6^{\circ}(\varrho=.456, p<.001)$ y $8^{\circ}(\varrho=.222, p=.035)$. Sin embargo, no se pudo comprobar una asociación significativa entre los alumnos varones $(\varrho=.072, p=.322)$, ni entre los tipos de centro, ni en los escolares de $4^{\circ}$ curso $(\varrho=.169, p=.080)$. 
Tabla 5. Correlaciones entre vocabulario y comprensión lectora.

\begin{tabular}{|l|l|c|c|c|}
\hline \multirow{3}{*}{ Género } & & N & Correlación $^{\mathbf{a}}$ & p-valor \\
\hline \multirow{3}{*}{ Colegios } & Niños & 192 & .072 & .322 \\
\cline { 2 - 5 } & Niñas & 212 & .227 & .001 \\
\hline \multirow{2}{*}{ Ciclos } & Privado & 175 & .006 & .935 \\
\cline { 2 - 5 } & Concertado & 118 & .115 & .214 \\
\cline { 2 - 5 } & Público & 111 & .145 & .129 \\
\hline \multirow{4}{*}{ Niveles } & $1^{\circ}$ & 206 & .135 & .054 \\
\cline { 2 - 5 } & $2^{\circ}$ & 198 & .374 & .001 \\
\hline & $2^{\circ}$ básico & 108 & .290 & .004 \\
\cline { 2 - 5 } & $4^{\circ}$ básico & 108 & .169 & .001 \\
\cline { 2 - 5 } & $6^{\circ}$ básico & 90 & .456 & .002 \\
\cline { 2 - 5 } & $8^{\circ}$ básico & 404 & .222 & .155 \\
\hline
\end{tabular}

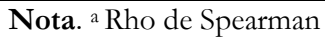

No obstante, para estudiar la asociación entre los resultados obtenidos en el test de vocabulario y en el test de comprensión lectora, y obtener deducciones concluyentes, se aplicó una regresión múltiple (multivariable), ajustada por el género, el curso y el colegio para cada uno de los ciclos por separado. A su vez los resultados se ajustaron con los datos obtenidos del test de Raven con el propósito de poder obtener con mayor precisión y ajuste la asociación entre ambas variables. Se ajustaron modelos multivariables mixtos con efecto de nivel por ciclo, curso o colegio y un modelo multivariable sin la inclusión del efecto de nivel, para comprobar su bondad de ajuste. El modelo con mejor bondad de ajuste fue el modelo sin nivel por curso o colegio y con efectos fijos por género, curso, colegio, clasificación Raven y clasificación TEVIR (BIC 1811,81) en comparación con la bondad de ajustes de modelos alternativos que superaban los valores de 1827,97.

Los resultados obtenidos se muestran en la Tabla 6, donde puede observarse una asociación significativa entre las variables vocabulario y comprensión lectora. El alumnado con retraso en vocabulario logró un menor porcentaje de aciertos que los escolares incluidos en las categorías bueno o sobresaliente. En particular, el porcentaje de acierto en la prueba de comprensión lectora en niños con retraso en vocabulario fue aproximadamente un $7 \%$ menor (diferencia ajustada con $p=.004$ ). No se apreciaron diferencias significativas en comprensión lectora entre los niños que mostraban un nivel de vocabulario normal y bueno/sobresaliente ( $p=.429)$, observando que una vez alcanzados valores normales en vocabulario, una mejora de estos no influía significativamente en una mejora directa de la comprensión. Sin embargo, cuando los valores de vocabulario no son normales, una mejora en el vocabulario implica una mejora directa en la comprensión lectora. En este sentido, para realizar una interpretación más sustantiva de los resultados obtenidos en la investigación, se calculó el tamaño del efecto, que cuantifica la magnitud de las diferencias encontradas en el estudio, y se demostró que entre el nivel de vocabulario 
retraso y bueno y sobresaliente existía una magnitud del efecto mediana (.49), y pequeña (.02) entre el nivel de vocabulario normal y bueno y sobresaliente.

Asimismo, se observó que los estudiantes con mayor nivel de inteligencia lograban de media un $8 \%$ más de aciertos que los que poseían una inteligencia inferior al término medio $(\mathrm{p}=.004)$. Los resultados del test de Raven revelaron que el 50\% de los escolares se encontraban en el Rango III, un 31,9\% en el Rango II y un 18,1\% en el Rango IV. Solo se observaron diferencias significativas entre los centros educativos $(p<.001)$ y no se advirtieron por razón de género, ciclo o nivel educativo ( $p>.05)$. Calculado el tamaño del efecto se observó que éste era moderado (.37) entre el nivel de inteligencia inferior al término medio y superior al término medio, y pequeño (.18) entre nivel de inteligencia término medio y superior. No se detectaron entre el alumnado participante diferencias significativas por género, ciclo o nivel educativo. En cambio, se encontraron diferencias significativas entre los centros escolares $(p<.001)$. Por otro lado, se constató una clara diferencia entre los alumnos de segundo y cuarto con respecto a los de segundo ciclo. En particular, los escolares de segundo y cuarto obtienen un $19 \%$ y un $9 \%$ más de aciertos, respectivamente, que los estudiantes de octavo $(p<.001)$, siendo las diferencias entre los alumnos de segundo y cuarto de un $10 \%$ aproximadamente $(p<.01)$. Por el contrario, entre los escolares de sexto y octavo, apenas se diferencian .03\% $(p=.991)$. Se detectó una magnitud del efecto grande (.81) entre los resultados obtenidos por el alumnado de $2^{\circ}$ y $8^{\circ}$ básico, un efecto mediano (.5) entre $4^{\circ}$ y $8^{\circ}$ curso, y pequeño (.08) entre $6^{\circ}$ y $8^{\circ}$ básico.

Ajustando los resultados por colegio, se observaron diferencias significativas entre los colegios privado y concertado con respecto al público, obteniéndose en cada uno de ellos un 19\% y un 8\% más de aciertos, respectivamente, que en el colegio público $(p<.01)$. De igual modo, en el colegio privado se observó aproximadamente un 11\% significativamente más de aciertos que en el centro concertado. El tamaño del efecto entre los centros privado y público es alto (.67), y moderado (.34) entre el colegio concertado y el público. 
Tabla 6. Resultados de la regresión múltiple (multivariable).

\begin{tabular}{|c|c|c|c|c|c|c|c|c|}
\hline & Parámetro & $\begin{array}{l}\text { Estima- } \\
\text { ción } \\
(\beta)\end{array}$ & $\begin{array}{l}\text { Error } \\
\text { típico }\end{array}$ & g.1. & $\mathbf{t}$ & $\begin{array}{l}\mathrm{p}- \\
\text { valor }\end{array}$ & $\begin{array}{l}\text { Intervalo } \\
95 \% \\
\text { Inferior }\end{array}$ & $\begin{array}{l}\text { e confianza } \\
\text { Superior }\end{array}$ \\
\hline $\begin{array}{l}\text { Géner } \\
\text { o }\end{array}$ & Niño $v s$. Niña & -1.297 & 1.859 & 393 & -0.698 & .486 & -4.952 & 2.357 \\
\hline \multirow{3}{*}{$\begin{array}{l}\text { Nivel } \\
\text { escolar }\end{array}$} & $2^{\circ} v s .8^{\circ}$ & 19.318 & 2.691 & 393 & 7.179 & $<.001$ & 14.028 & 24.608 \\
\hline & $4^{\circ} v s .8^{\circ}$ & 9.270 & 2.610 & 393 & 3.552 & $<.001$ & 4.139 & 14.401 \\
\hline & $6^{\circ} v s .8^{\circ}$ & 0.032 & 2.688 & 393 & 0.012 & .991 & -5.254 & 5.318 \\
\hline \multirow[t]{2}{*}{$\begin{array}{l}\text { Raven } \\
\text { (Inteli- } \\
\text { gencia) }\end{array}$} & $\begin{array}{l}\text { Inferior al } \\
\text { término } \\
\text { medio } v s \text {. } \\
\text { Superior }\end{array}$ & -8.124 & 2.800 & 393 & -2.902 & .004 & -13.629 & -2.620 \\
\hline & $\begin{array}{l}\text { Término } \\
\text { medio } v s . \\
\text { Superior }\end{array}$ & -2.937 & 2.096 & 393 & -1.401 & .162 & -7.058 & 1.185 \\
\hline \multirow{2}{*}{$\begin{array}{l}\text { TEVI } \\
\text { (Nivel } \\
\text { de } \\
\text { Voca- } \\
\text { bulario } \\
\text { ) }\end{array}$} & $\begin{array}{l}\text { Retraso vs. } \\
\text { Bueno/Sobres } \\
\text { a-liente }\end{array}$ & -7.436 & 2.556 & 393 & -2.909 & .004 & -12.461 & -2.411 \\
\hline & $\begin{array}{l}\text { Normal } v s . \\
\text { Bueno/Sobres } \\
\text { a-liente }\end{array}$ & -2.223 & 2.806 & 393 & -0.792 & .429 & -7.740 & 3.293 \\
\hline \multirow{2}{*}{$\begin{array}{l}\text { Tipo } \\
\text { de } \\
\text { colegio }\end{array}$} & $\begin{array}{l}\text { Privado } v s . \\
\text { Público }\end{array}$ & 18.837 & 2.357 & 393 & 7.993 & $<.001$ & 14.204 & 23.470 \\
\hline & $\begin{array}{l}\text { Concertado } v s . \\
\text { Público }\end{array}$ & 7.993 & 2.491 & 393 & 3.208 & .001 & 3.095 & 12.891 \\
\hline
\end{tabular}

\section{DISCUSIÓN Y CONCLUSIONES}

Como se ha señalado, el propósito central de este estudio fue describir y analizar la asociación entre vocabulario y comprensión lectora en escolares chilenos de educación básica, teniendo en cuenta el género, la capacidad cognitiva, el nivel escolar, el ciclo y el tipo de centro. Inicialmente, los datos han revelado que los estudiantes de primer ciclo lograban mayores niveles en comprensión lectora que los del segundo, con independencia del género. Este dato no concuerda con los hallazgos de otras investigaciones previas en las que se admitía una mayor competencia de las niñas (Jiménez, 2010), información que se corrobora en los últimos resultados de lectura publicados por el SIMCE (2018). Sin embargo, el hallazgo es consistente con los estudios que han demostrado que los alumnos de cursos superiores no necesariamente alcanzan puntuaciones más altas en comprensión lectora (Hirsch, 2007; Lara et al., 2010; De Mier, Borzone, Sánchez \& Benítez, 2013). Las principales diferencias se observaron entre $2^{\circ}$ y $8^{\circ}$ básico y las menores entre $6^{\circ}$ y $8^{\circ}$ curso.

No obstante, aunque en los cursos superiores se observó una mayor homogeneidad, los estudiantes no progresaban como cabría esperar. Este aporte coincide con los estudios que muestran cierta tendencia de los estudiantes a encontrar una dificultad lingüística progresiva en tareas de comprensión lectora (Alliende et al., 
2012; Rodríguez, Calderón, Leal \& Arias, 2016), antecedente que no debería ser interpretado como una menor capacidad comprensiva de los escolares de cursos superiores, sino como una consecuencia atribuible a la mayor complejidad de las pruebas a las que éstos se enfrentan.

Los resultados también han puesto de manifiesto que la escolarización en un determinado centro escolar incide de forma decisiva en la comprensión lectora. De hecho, se han apreciado diferencias significativas entre el alumnado según el tipo de colegio al que pertenecen. Los escolares del centro privado alcanzaron mejores puntuaciones que los del colegio concertado, los cuales superan, a su vez, a los estudiantes del colegio público. En todo caso, las mayores diferencias se observaron entre el centro privado y el público. Este dato es consistente con los estudios que confirman la existencia de marcadas diferencias en el desempeño lector de los escolares, según su escolarización en centros públicos o privados (Urquijo, 2009). Además, si se tiene en cuenta que el NSE del alumnado difiere en los tres centros, podría aventurarse que las condiciones socioeconómicas redundan potencialmente en la comprensión, hecho que en otras investigaciones se ha considerado como uno de los factores más determinante (Suárez et al., 2010; Morales, 2011; Cuadro \& Balbi, 2012; Fonseca et al., 2014; Urquijo et al., 2015).

En cuanto al desarrollo léxico, se apreciaron diferencias significativas entre los escolares por curso, ciclo y tipo de colegio. Si bien la tendencia ahora es inversa a la de comprensión lectora, por cuanto se constató que a medida que aumentaba el grado escolar se incrementaba el nivel de vocabulario de los estudiantes, excepto en $8^{\circ}$ curso. Investigaciones previas ya cuestionaron la supuesta progresión léxica de los estudiantes que promocionaban de curso (Hart \& Risley, 2003; Suárez et al., 2010). No obstante, el incremento del vocabulario de los escolares conforme avanzan de curso está suficientemente documentado (White, Graves \& Slater, 1990; Pavez, Rojas, Rojas \& Zambra, 2016).

Teniendo en cuenta el tipo de colegio, en cuanto al nivel de vocabulario, se observó que los estudiantes del centro privado aventajaban a los escolares de los centros público y concertado, debido tal vez a su mayor NSE. La variable género tampoco fue determinante, al no observarse diferencias léxicas significativas entre niños y niñas. Como han señalado otros investigadores, aunque la comprensión lectora no dependa solamente del nivel de vocabulario del estudiante (Morales, 2011; Vieiro \& Amboage, 2015), los niños con déficit en el vocabulario muestran problemas para comprender el sentido de un texto (Lara et al., 2010). De forma recíproca, la ausencia de destrezas lectoras en los niños también incide de forma negativa en el desarrollo del vocabulario (Catts \& Kamhi, 2005).

Considerando la muestra global, el análisis bivariante desveló una asociación significativa entre el vocabulario y la comprensión lectora, que se visibilizó 
especialmente entre las alumnas y en los niveles de $2^{\circ}, 6^{\circ}$ y $8^{\circ}$ básicos. Estos hallazgos corroboran la sólida relación entre el léxico y la comprensión lectora, al considerar al vocabulario como un predictor consistente de ésta (Beck, Perfetti \& McKeown, 1982; Verhoeven \& Perfetti, 2011; Moghadam et al., 2012; Protopapas, Mouzaki, Sideridis, Kotsolakou \& Simos, 2013; López \& Rubio, 2014; Perfetti \& Stafura, 2014; Riffo, Reyes, Novoa, Véliz \& Castro, 2014; Villalonga et al., 2014; Riffo et al., 2018). Sin embargo, se apreció una baja correlación en los alumnos varones, los estudiantes de $4^{\circ}$ curso y los centros educativos. Este descubrimiento es consistente con los hallazgos de otras investigaciones en las que el vocabulario no fue tan decisivo como se esperaba para la comprensión lectora (Muter et al., 2004; Salvador et al., 2007).

El análisis multivariable confirmó la existencia de una asociación significativa entre el vocabulario, según la categorización de los escolares, y las puntuaciones obtenidas en comprensión lectora. Los alumnos que mostraron retraso en vocabulario consiguieron menor porcentaje de aciertos que los alumnos que obtuvieron un nivel bueno o sobresaliente. Sin embargo, cuando los escolares no lograron un nivel de vocabulario normal, bueno o sobresaliente, un incremento léxico suponía una mejora directa de la comprensión lectora. Estos descubrimientos son coherentes con los resultados de estudios anteriores en los que se observó una alta correlación entre el vocabulario y la comprensión (Nation et al., 2010; González \& Castro, 2016). Pero no se puede ignorar que las mayores diferencias en cuanto al vocabulario se establecieron entre las categorías retraso y bueno o sobresaliente, y no entre las categorías normal y bueno o sobresaliente. Un requisito esencial del éxito lector, subrayan Castro, Mathiesen, Mora, Merino y Navarro (2012), lo representa el léxico, ya que cuanto más rico sea este más efectivos serán los procesos comprensivos. La comprensión depende, en buena medida, de la lectura de palabras y las diferencias en habilidades de comprensión pueden generarse en las desiguales destrezas del alumnado para enfrentarse a la lectura de éstas (Perfetti, 2010).

Equivalentemente se demostró que los escolares con mayor capacidad cognitiva lograban un mejor rendimiento lector que los que poseían una inteligencia inferior al término medio, como ya observaran otros investigadores (Castro et al., 2012; Bohórquez et al., 2014), si bien las diferencias fueron moderadas. Asimismo, se observó que los escolares de primer ciclo aventajaban en tareas de comprensión lectora a los estudiantes de segundo, tal vez por la simplicidad de los textos a los que se enfrentan los alumnos de los primeros niveles y la complejidad que encierran los textos en los cursos superiores (Hirsch, 2007). Igualmente, se ha corroborado el papel que juega la escolarización de los estudiantes en la comprensión. Los escolares pertenecientes a los colegios privado y concertado obtuvieron un mayor rendimiento lector que los del centro público, tal vez por la incidencia que el NSE pudiera ejercer sobre el alumnado (Fonseca et al., 2014; Urquijo et al., 2015), además de otras variables no analizadas en este estudio. 
En conclusión, los análisis realizados ponen de manifiesto que el vocabulario, el nivel escolar, la capacidad cognitiva de los estudiantes y el tipo de centro tienen incidencia directa en la comprensión lectora, sin que la variable género haya sido determinante en ningún caso. Sin embargo, la interpretación de los resultados de esta investigación debe hacerse a la luz de sus limitaciones. En primer lugar, se presentan datos de escolares de una sola zona geográfica, que impiden su generalización. En segundo lugar, aunque la regresión pueda ser considerada como indicadora de causalidad, los resultados poseen más un carácter correlacional que causal. En líneas futuras de estudio, y puesto que los resultados del análisis multivariable indicaron una tendencia no lineal entre los cursos, sería interesante analizar la posible tendencia que exista, ajustando modelos polinómicos o modelos generales aditivos multivariantes (GAM) a lo largo de los cursos para ajustar una curva de tendencia. Asimismo, se podría realizar una investigación más profunda mediante un análisis de ecuaciones estructurales. Igualmente, sería aconsejable realizar futuras investigaciones en las que se analicen otras variables (por ejemplo, métodos de lectura, metodologías docentes, etc.) que condicionan el aprendizaje lector, con muestras más amplias y de otras zonas geográficas y otros niveles escolares.

\section{REFERENCIAS BIBLIOGRÁFICAS}

Alliende, F. \& Condemarín, M. (2002). La lectura: Teoría, evaluación y desarrollo. Santiago de Chile: Andrés Bello.

Alliende, F., Condemarín, M. \& Milicic, N. (2012). Prueba CLP formas paralelas. Prueba de comprensión lectora de complejidad lingüistica progresiva. Santiago de Chile: Pontificia Universidad Católica de Chile.

Ato, M., López, J. J. \& Benavente, A. (2013). Un sistema de clasificación de los diseños de investigación en psicología. Anales de Psicología, 29(3), 1038-1059.

Beck, I. L., Perfetti, C. A. \& McKeown, M. G. (1982). Effects of long-term vocabulary instruction on lexical access and reading comprehension. Journal of Educational Psychology, 74(4), 506-521.

Bohórquez, L. F., Cabal, M. A. \& Quijano, M. C. (2014). La comprensión verbal y la lectura en niños con y sin retraso lector. Pensamiento Psicológico, 12(1) 169-182.

Canet, L., Burin, D., Andrés, M. L. \& Urquijo, S. (2013). Perfil cognitivo de niños con rendimientos bajos en comprensión lectora. Anales de Psicología, 29(3), 9961005. DOI: $10.6018 /$ analesps.29.3.138221

Carrillo, M. \& Alegría, J. (2015). Mecanismos de identificación de palabras en niños disléxicos en español: ¿Existen subtipos? Ciencias Psicológicas, 3, 135-152. 
Castro, G. G., Mathiesen, M. E., Mora, O., Merino, J. M. \& Navarro, G. (2012). Habilidades lingüísticas y rendimiento académico en escolares talentosos. Revista CES Psicología, 5(2), 40-55.

Catts, H. W. \& Kamhi, A. G. (2005). The connections between lan-guage and reading disabilities. Mahwah NJ: LawrenceErlbaum.

Cepeda, M., Granada, M. \& Pomes, M. (2014). Disponibilidad léxica en estudiantes de primero básico. Literatura y Lingüistica, 30, 166-181.

Coelho, C. L. G. \& Correa, J. (2017). Compreensão de leitura: Habilidades cognitivas e tipos de texto. Revista Psico, 48(1), 40-49.

Cuadro, A. \& Balbi, A. (2012). Las diferencias socioeconómicas y la lectura: Claves para analizar los resultados de las evaluaciones PISA. Revista Neuropsicología Latinoamericana, 4(1), 57-64.

De Mier, M. V., Borzone, A. M., Sánchez, V. S. \& Benítez, M. E. (2013). Habilidades de comprensión y factores textuales en los primeros grados. Revista Peruana de Psicología y Trabajo Social, 2(1), 89-106.

Echeverría, M., Herrera, M. \& Segure, J. (2002). TEVI- R, Test de vocabulario en imágenes. Chile: Universidad de Concepción.

Fonseca, L., Pujals, M., Lasala, E., Lagomarsino, I. Migliardo, G., Aldrey, A., Buonsanti, L. \& Barreyro, J. P. (2014). Desarrollo de habilidades de comprensión lectora en niños de escuelas de distintos sectores socioeconómicos. Revista Neuropsicología Latinoamericana, 6(1), 41-50.

González, K. \& Castro, A. M. (2016). Factores cognitivos asociados a la comprensión lectora en niños cubanos de sexto grado. Integración Académica en Psicología, 4(12), 96-105.

Hart, B. \& Risley, T. D. (2003). The early catastrophe. American Educator, 27(1), 4-9.

Hirsch, E. D. (2003). Reading comprehension requires knowledge-of words and the world. American Educator, 27(1), 10-13.

Hirsch, E. D. (2007). La comprensión lectora requiere conocimiento de vocabulario y del mundo: Hallazgos científicos sobre el bajón de cuarto grado y el estancamiento en los puntajes nacionales de comprensión. Estudios Públicos, 108, 229-252. 
Jiménez, E. (2010). El factor género en el proceso de adquisición de lenguas: Revisión crítica de los estudios interdisciplinares. Lingüística en la Red [en línea]. Disponible en: http://www.linred.es/articulos_pdf/LR_articulo_30042010.pdf?

Lara, M. F., Gómez, A. M., García, M., Guerrero, Y. \& Niño, L. (2010). Relaciones entre las dificultades del lenguaje oral a los 5 y 6 años y los procesos de lectura a los 8 y 9 años. Revista Facultad de Medicina, 58(3), 191-203.

Lonigan, C., Burgess, S. \& Anthony, J. (2000). Development of emergent literacy ans early reading skills in preschool children: Evidence a lantent-variable longitudinal study. Developmental Psychology, 36(5), 596-613.

López, R. \& Rubio, S. (2014). ¿Qué variables determinan el nivel lector de un alumno en el segundo ciclo de Educación Primaria y cuál es su valor diagnóstico? Revista Iberoamericana de Diagnóstico y Evaluación - e Avaliação Psicológica, 1(37), 31 52.

McNamara, D. S. \& Magliano, J. P. (2009). Towards a comprehensive model of comprehension. En B. Ross (Ed.), The psychology of learning and motivation (pp. 297-384). Nueva York: Academic Press.

Moghadam, S. H., Zainal, Z. \& Ghaderpour, M. (2012). A review on the important role of vocabulary Knowledge in reading comprehension performance. Procedia - Social and Behavioral Sciences, 66, 555-563.

Molina, S. (2008). Psicopedagogía de la lengua escrita: Lectura. Madrid: EOS.

Morales, S. (2011). Variables socioculturales y cognitivas en el desarrollo de la comprensión de lectura en Lima, Perú. Revista Peruana de Investigación Educativa, 3, 96-129.

Muter, V., Hulme, Ch., Snowling, M. J. \& Stevenson, J. (2004). Phonemes, rimes, vocabulary, and grammatical skills as foundations of early reading development: evidence from a longitudinal study. Developmental Psychology, 40(5), 665-681.

Nagy, W. E. \& Scott, J. A. (2000). Vocabulary processes. En M. L. Kamil, P. Mosenthal, P. D. Pearson \& R. Barr (Eds.), Handbook of reading research (pp. 269-284). Mahwah, NJ: Erlbaum.

Nation, K., Cocksey, J., Taylor, J. S. \& Bishop, D. V. (2010). A longitudinal investigation of early reading and language skills in children with poor reading comprehension. Journal of Child Psychology and Psychiatry, 51(9), 1031-1309. 
Nation, K. \& Snowling, M. J. (1998). Semantic processing and the development of word recognition skills: Evidence from children with reading comprehension difficulties. Journal of Memory and Language, 39(1), 85-101.

Nation, K. \& Snowling, M. J. (2004). Beyond phonological skills: Broader language skills contribute to the development of reading. Journal of Research in Reading, 27(4), 342-356.

Oakhill, J. V. \& Cain, K. (2012). The precursors of reading ability in young readers: Evidence from a four-year longitudinal study. Scientific Studies of Reading, 16(2), 91-121

Pavez, A., Rojas, P., Rojas, F. \& Zambra, N. (2016). Habilidades semánticas y rendimiento académico en escolares de $2^{\circ}$ y $4^{\circ}$ año básico. Revista Chilena de Fonoaudiología, 15, 1-12.

Perfetti, C. (2007). Reading ability: Lexical quality to comprehension. Scientific Studies of Reading, 11, 357-383.

Perfetti, C. (2010). Decoding, vocabulary and comprehension. The golden triangle of reading skill. En M. McKeown \& L. Kucan (Eds.), Bringing Reading Research to Life (pp. 291-303). Nueva York: The Guilford Press.

Perfetti, C. \& Stafura, J. (2014). Word knowledge in a theory of reading comprehension. Scientific Studies of Reading, 18(1), 22-37.

Protopapas, A., Mouzaki, A., Sideridis, G. D., Kotsolakou, A. \& Simos, P. G. (2013). The role of vocabulary in the context of the simple view of reading. Reading $\&$ Writing Quarterly, 29(2), 168-202.

Raven, J., Court, J. \& Raven, J. (2014). Test de Matrices Progresivas: Escala coloreada, general y avanzada. Buenos Aires: Paidós.

Ricketts, J., Nation, K. \& Bishop, D. V. M. (2007). Vocabulary is important for some, but not all reading skills. Scientific Studies of Reading, 11(3), 235-257.

Riffo, B., Reyes, F., Novoa, A., Véliz, N. \& Castro, G. (2014). Competencia léxica, comprensión lectora y rendimiento académico en estudiantes de enseñanza media. Literatura y Lingïistica, 30, 165-180.

Riffo, B., Caro, N. \& Sáez, K. (2018). Conciencia lingüística, lectura en voz alta y comprensión lectora. Revista de Lingü̈stica Teórica y Aplicada, 56(2), 175-198. 
Rodríguez, B. A., Calderón, M. E., Leal, M. H. \& Arias, N. (2016). Uso de estrategias metacomprensivas para el fortalecimiento de la comprensión lectora en estudiantes de segundo ciclo de un colegio oficial en Bogotá, Colombia. Revista Folios, 44, 93-108.

Salvador, F., Gallego, J. L. \& Mieres, C. (2007). Habilidades lingüísticas y comprensión lectora. Una investigación empírica. Bordón 59(1), 153-166 [en línea]. Disponible en: https://dialnet.unirioja.es/servlet/articulo?codigo $=2533523$

Schelling, G., Aarnoutse, C. \& Leeuwe, J. V. (2006). Third-grader's think-aloud protocols: Types of reading activities in reading an expository text. Learning and Instruction, 16(6), 549-568.

SIMCE (2018). Resultados nacionales. Santiago de Chile: Ministerio de Educación. https://www.agenciaeducacion.cl/evaluaciones/que-es-el-simce/

Sousa, L. B. \& Hübner, L. C. (2017). La relación entre desempeño en comprensión lectora y factores socioeconómicos en escuelas públicas del sur de Brasil. Fórum Lingüistico, 14(2), 2044-2060.

Suárez, A., Moreno, J. M. \& Godoy, M. J. (2010). Vocabulario y comprensión lectora: Algo más que causa y efecto. Álabe. Revista de Investigación sobre Lectura y Escritura, 1, 1-18.

Swart, N. M., Muijselaar, M. M., Steenbeek, E. G., Droop, M., de Jong, P. F. \& Verhoeven, L. (2017). Cognitive precursors of the developmental relation between lexical quality and reading comprehension in the intermediate elementary grades. Learning and Individual Differences, 59, 43-54.

Urquijo, S. (2009). Aprendizaje de la lectura. Diferencias entre escuelas de gestión pública y de gestión privada. Revista Evaluar, 9, 19-34.

Urquijo, S., García, A. \& Fernandes, D. (2015). Relación entre aprendizaje de la lectura y nivel socioeconómico en niños argentinos. Avances en Psicología Latinoamericana, 33(2), 303-318.

Verhoeven, L. \& Perfetti, C. A. (2011). Introduction to this special issue: Vocabulary growth and reading skill. Scientific Studies of Reading, 15(1), 1-7.

Vieiro, P. \& Amboage, I. (2015). Relación entre habilidades de lectura de palabras y comprensión lectora. Revista de Investigación en Logopedia, 1, 1-21.

Villalonga, M. M., Padilla, C. \& Burin, D. (2014). Relaciones entre decodificación, conocimiento léxico-semántico e inferencias en niños de escolaridad primaria. Interdisciplinaria, 31(2), 259-274. 
White, T. G., Graves, M. F. \& Slater, W. H. (1990). Growth of reading vocabulary in diverse elementary schools: Decoding and word meaning. Journal of Educational Psychology, 82(2), 281-290.

Willems, G., Jansma, B., Blomert, B. \& Vaessen, A. (2016). Cognitive and familial risk evidence converged: A data-driven identification of distinct and homogeneous subtypes within the heterogeneous sample of reading disabled children. Research in Developmental Disabilities, 53-54, 213-231. 